\title{
Co-expression network analysis and genetic algorithms for gene prioritization in preeclampsia
}

\author{
Eduardo Tejera', João Bernardes ${ }^{2,3}$ and Irene Rebelo ${ }^{4,5 *}$
}

\begin{abstract}
Background: In this study, we explored the gene prioritization in preeclampsia, combining co-expression network analysis and genetic algorithms optimization approaches. We analysed five public projects obtaining 1,146 significant genes after cross-platform and processing of 81 and 149 microarrays in preeclamptic and normal conditions, respectively.

Methods: After co-expression network construction, modular and node analysis were performed using several approaches. Moreover, genetic algorithms were also applied in combination with the nearest neighbour and discriminant analysis classification methods.

Results: Significant differences were found in the genes connectivity distribution, both in normal and preeclampsia conditions pointing to the need and importance of examining connectivity alongside expression for prioritization. We discuss the global as well as intra-modular connectivity for hubs detection and also the utility of genetic algorithms in combination with the network information. FLT1, LEP, INHA and ENG genes were identified according to the literature, however, we also found other genes as FLNB, INHBA, NDRG1 and LYN highly significant but underexplored during normal pregnancy or preeclampsia.

Conclusions: Weighted genes co-expression network analysis reveals a similar distribution along the modules detected both in normal and preeclampsia conditions. However, major differences were obtained by analysing the nodes connectivity. All models obtained by genetic algorithm procedures were consistent with a correct classification, higher than 90\%, restricting to 30 variables in both classification methods applied. Combining the two methods we identified well known genes related to preeclampsia, but also lead us to propose new candidates poorly explored or completely unknown in the pathogenesis of preeclampsia, which may have to be validated experimentally.
\end{abstract}

\section{Background}

Preeclampsia remains a leading cause of maternal/fetal mortality and morbidity associated with gestational hypertension and proteinuria. The underlying mechanism and preventive treatment $[1,2]$ remain unknown and therefore, it is still known as the "disease of theories" [3]. Due to possible multifactorial causes involved [1,2,4], an increase in "omics" experimental approaches is noted, generating a large amount of information, not always integrated or analysed by recent methodologies.

\footnotetext{
* Correspondence: irebelo@ff.up.pt

${ }^{4}$ Laboratory of Biochemistry, Department of Biological Sciences, Faculty of Pharmacy, University of Porto, Porto, Portugal

${ }^{5}$ Institute for Molecular and Cell Biology (IBMC), University of Porto, Portugal, Porto, Portugal

Full list of author information is available at the end of the article
}

Some bioinformatics analysis were performed on specific microarray assays [5-7], and our group has recently carried out an extensive review of related data, processing multiple microarrays combined with text mining tools that led to the identification of several specific genes [8].

In this work, we present a different strategy focused on gene prioritization by co-expression network analysis and genetic algorithms optimization. We also increase the number of microarrays processed.

\section{Methods \\ Microarray processing}

Experimental microarray data comparing normal $(\mathrm{N})$ and preeclamptic pregnancies (PRE) was obtained analysing the Gene Expression Omnibus (GEO) and ArrayExpress

\section{Biomed Central}

(c) 2013 Tejera et al.; licensee BioMed Central Ltd. This is an open access article distributed under the terms of the Creative Commons Attribution License (http://creativecommons.org/licenses/by/2.0), which permits unrestricted use, distribution, and reproduction in any medium, provided the original work is properly cited. 
databases $[9,10]$. Only the studies comprising more than 10 subjects (by groups) were included (Table 1 ).

Table 1 shows the GEO and Array Express data sources, references, and additional information used in the study. Each microarray was processed as follows: for Affymetrix platforms, the raw data was mas5 preprocessed and $\log 2$ transformed using affy package [15], in Bioconductor [16]; for Ilumina platforms, batch correction, normalization and $\log 2$ transformation were performed using the lumi package [17], also in Bioconductor; finally, ABI Human platform was used as provided. The authors (GSE 10588, ABI platform) indicated that the arrays were quantile normalized and background correction was performed using ABI 1700 software, however, we extracted and processed the public data using GEOquery package [18] in Bioconductor.

In cross-platform microarray analysis the first step, after individual microarrays analysis is to combine the different probes. For this task usually a common identifier is used (i.e. entrez gene, unigene code) in order to obtain the common space across all platforms [19-21]. We mapped the arrays probes for the respective entrez gene ID through manual observation and also using the updated manufacturers annotation information (using R-packages: lumiHumanIDMapping and hgu133b.db [22,23]) for all platforms. Only genes common to all platforms (6816 genes) were used in the subsequent analysis. Genes with more than one probe were combined by averaging the intensity values using collapseRows and intersect the functions available in the WGCNA package [24,25]. The second normalization was performed in order to re-scale the intensity and also remove cross-platform batch effects using Combat function in SVA package [26]. The identification of genes with statistically different expression between $\mathrm{N}$ and PRE groups was performed using lmFit from Limma R-Package [27] and only genes with $\mathrm{p} \leq 0.05$ ( $\mathrm{n}=$ 1146 genes) were considered for co-expression networks construction.

\section{Co-expression network construction and analysis}

Genes differentiated ( $\mathrm{n}=1146$ genes) between $\mathrm{N}$ and PRE groups were used for weighted genes co-expression $(\mathrm{CoE})$ network construction in each group using

Table 1 General microarrays information

\begin{tabular}{llclll}
\hline Code & Database & \multicolumn{1}{c}{ Sample } & Method & Tissue & Ref. \\
\hline E-TABM-682 & Array express & 13(PRE), 58(N) & Illumina & Placenta & [11] \\
E-MEXP-1050 & Array express & 16(PRE), 17(N) & Affymetrix & Placenta & [12] \\
GSE25906 & GEO & 23(PRE), 37(N) & Illumina & Placenta & [13] \\
GSE14722 & GEO & 12(PRE), 11(N) & Affymetrix & Placenta & [14] \\
GSE10588 & GEO & 17(PRE), 26(N) & ABl Human & Placenta & [7] \\
\hline
\end{tabular}

Notes: All samples were collected for biopsy of placenta during childbirth. 2) In the GEO appear two platforms (GPL96, GPL97) but only GPL96 was used because a greater number of probes are shared with other platforms.
WGCNA package [24]. In the weighted genes coexpression network the nodes represent genes and the edges represent the connection strength which corresponds essentially to a weighted adjacency matrix (A) with elements $\alpha_{i, j}=\left|\operatorname{cor}\left(x_{i}, x_{j}\right)\right|^{\beta}$ where $x_{i}$ and $x_{j}$ are the expression profiles of genes $i$ and $j$. This method considers a continuous $([0,1]$ interval) instead of discrete adjacency matrix which proved to be highly robust with respect to the $\beta$ parameter variation.

In this study, we selected $\beta=6$, following the scale-free topology criterion proposed by Zhang and Horvath using the pickSoftThreshold function in WGCNA (data not shown) [28]. Once defined the adjacency matrix for each group (normal and PRE), the co-expression matrix (CoN and CoP, respectively) and the topological overlap matrix (TOM) were obtained (Figure 1). The topological overlap matrix (TOM) is the central starting point for network modules detection and analysis and each element $\left(\omega_{\mathrm{ij}}\right)$ represents a measure of similarity between two nodes in the same network.

Further analyses were divided into main branch (Figure 1): a) modular (inter and intra-modules) analysis and b) genes (nodes) analysis.

\section{Modules analysis}

The modules were detected using the Dynamic Tree Cut algorithm [29] with cutreeDynamic function in WGCNA package and defining the deep split $=3$ and cutting height corresponding to the 99th percentile and the maximum of the joining heights on the dendrograms. In each module, the node connectivity and the node intramodular connectivity were calculated. This means, the node connectivity is basically defined as the sum of the weights of all edges connected to it $\left(k_{i}=\sum_{j} \alpha_{i, j}\right)$, however, when a module is defined each gene is now linked with a specific subgroup of neighbours and therefore connectivity will change as a consequence of reduction of the number of neighbours, therefore, in a network with $\mathrm{m}=1,2, \ldots, \mathrm{M}$ modules and where each of these modules has $N_{m}$ nodes, the intra-modular connectivity $\left(k_{i}^{m}\right)$ is

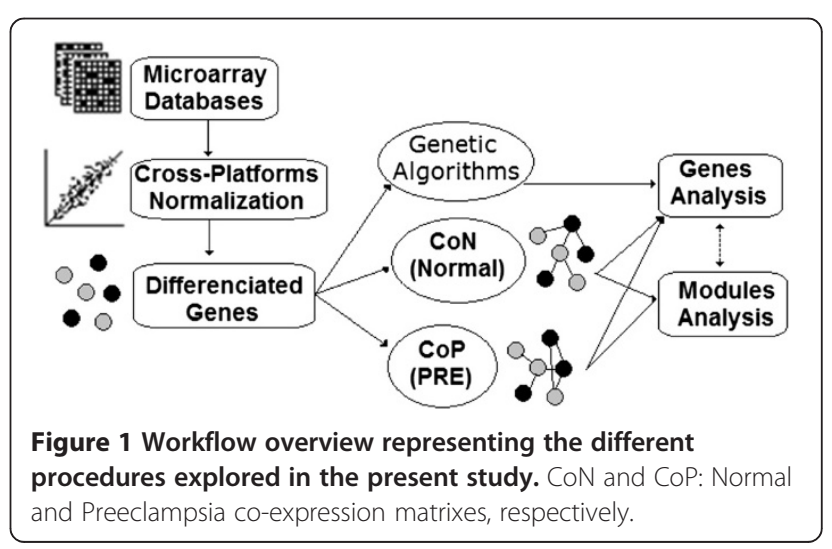


defined as $k_{i}^{m}=\sum_{j}^{N_{m}} a_{i, j}$, this means, the sum of the weights of all edges connected to node $i$ in module $m$.

We also compared the modules obtained for each network ( $\mathrm{CoN}$ and $\mathrm{CoP})$, using the Fisher's exact test, where we basically analysed the number of common genes between modules. Therefore, the modules (between the two conditions), with an increased number of shared genes tend to be more unspecific. Combined analysis of intra-modular connectivity and modules comparison could provide a better identification and description of each gene in the network modules, and therefore the identification of potential network hubs.

\section{Nodes analysis}

Even when the module analysis above could provide a deep inside in the network hubs in identifying PRE specific related genes, we are interested in a direct comparison between nodes (genes) in both networks. The weighted adjacency matrix represents a complete graph because each element of the network is connected (even with very low strength), moreover, both the network have the same number of nodes and edges, so if connectivity environment of a gene $i$ is similar in both network then the genes are also similar in both physiological states. Consequently, we define a distance measure of gene $i$ between two networks ( $\mathrm{CoN}$ and $\mathrm{CoP})$ as follows:

$$
\mathrm{KDist}_{i}=\frac{1}{k_{i}^{C o P}+k_{i}^{C o N}} \sqrt{\sum_{j=1}^{N}\left(\alpha_{i, j}^{C o P}-\alpha_{i, j}^{C o N}\right)^{2}}
$$

The highest values of KDist correspond to genes with very different connectivity environments and therefore more likely to be a significant gene in the PRE condition. Similar procedures were implemented by other authors, using the node degree obtained in binary adjacency matrix and counting the number of common edges $[30,31]$. To select a group with increased distance values, we need to identify a distance cut-off. The cut-off distance was selected for comparison with 1000 randomized network as follows: for each network ( $\mathrm{CoN}$ and CoP) 1000 network were obtained by random permutation of the original edges strengths $\left(a_{i, j}\right)$. Among all networks (randomized N and PRE) the KDist is computed followed by counting the number of nodes with distances higher than a predefined percent (cut-off value) of the maximum distance value. The selected numbers of genes for the different cut-off values were compared using t-test (similar strategy was followed in $[30,31]$ ).

Gene ontology and metabolic pathway enrichment analysis The gene ontology and pathways enrichment analysis were performed using DAVID bioinformatics resource v6.7 [32], exploiting the well know databases: gene Ontology and KEGG databases. Complete enrichment analyses of each of the network modules will considerable increase the length of the presented work and therefore we will present only the results obtained for specific and relevant modules.

\section{Genetic algorithm optimization in genes selection}

These procedures can also be seen as a node centred analysis, but considering exclusively expression values and not a network structure. The general idea is to identify genes (combination of them) that maximize the differentiation between $\mathrm{N}$ and PRE groups. In this context, we can apply a combination of genetic algorithm (GA) optimization with two widely used classification methods: Nearest Neighbour (GANN) and Discriminant Analysis (GADA). We used the Euclidian as the distance metric in the nearest neighbour algorithm and the linear discriminant function in the discriminant analysis. It is important to realize that other metrics can be used in both the nearest neighbour and discriminant analysis and probably will lead to different results, however, find the "best" strategy is not an objective of the present study and probably will depend on particular classification/data problem.

The GA was run 25 times with different initial populations and each of the final models was used in further analysis. The GA initial parameters were: 1,000 generations, the initial population of 100 chromosomes and a cross-over and mutation probability of 0.7 and 0.3 , respectively. The maximum number of selected genes was restricted to 30. The criterion for model selection was the leave-one-out (LOO) cross-validation procedure and therefore, for each algorithm, we have a set of 25 models and the error estimated by the LOO, respectively.

The 25 models obtained in each algorithm procedure (a total of $2 \times 25$ models and a maximum possibility of $25 \times 30$ different genes, by procedure) do not comprise the same genes but a space of them. However, some genes are frequently present across the models and therefore may be of specific interest in further considerations. We also cross-analysed the genes space obtained by the different GA procedures with the respective gene location in the network modules and also the relationship with KDist leading to integrated information and facilitates the interpretation.

\section{Results}

The correlation between the mean ranked expression, as well as the mean ranked connectivity between $\mathrm{N}$ and PRE groups shows a higher correlation for the expression instead of connectivity, even when both are statistically significant (Figure 2 ).

The average nodes degree in each network were (mean \pm standard error): $1.57 \pm 0.05(\mathrm{~N})$ and $32.7 \pm 0.19$ (PRE), indicating that the correlation between the gene 

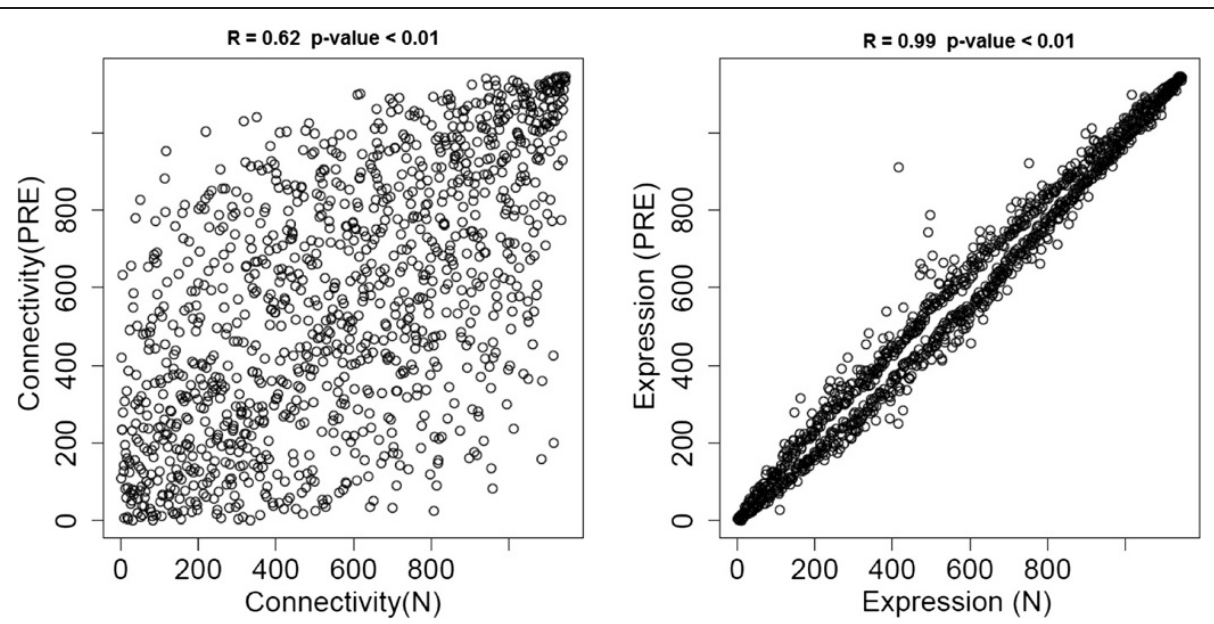

Figure 2 Left: Mean ranked expression comparison; Right: Mean ranked connectivity comparison between Normal and PRE group.

expression in PRE is increased. This means that the interconnectivity between genes is higher on PRE over N.

Using the Dynamic Tree Cut method, 8 and 7 modules were identified in $\mathrm{CoP}$ and $\mathrm{CoN}$, respectively (Figure 3) marked as follows: CoP) MP1 (53 genes), MP2 (203 genes), MP3 (186 genes), MP4 (104 genes), MP5 (52 genes), MP6 (84 genes), MP7 (310 genes) and MP8 (154 genes); CoN) MN1 (225 genes), MN2 (141 genes), MN3 (78 genes) MN4 (83 genes), MN5 (77 genes), MN6 (410 genes) and MN7 (132 genes). The MN4 (grey) correspond to those genes that are not grouped in any particular community.

The comparative enrichment module analysis reveals that all modules have a certain overlap between $\mathrm{N}$ and PRE group (Figure 4 Left) to some degree, suggesting that the genes are grouped in a similar fashion between the two conditions. Furthermore, we note that the MP4 comprise genes with large differences in the expression values (mainly up-regulated), suggesting that this module could include genes of interest (at least in terms of expression). In fact, the gene ontologies and pathways enrichment analysis indicates that biological processes like ( $\mathrm{p}$-value $<$ 0.01): ovulation cycle, sexual differentiation, regulation of hormone levels and the erythrocyte differentiation are significantly enriched in the MP4 module, as well as metabolic pathway related with the GnRH signalling pathway that is closely related with the cytokine-cytokine receptors interactions. Hormonal modifications and cytokine signalling processes are highly relevant in the PRE (see Discussion).

Similar modules relevance can be considered for MP6 and MP8 because they comprise (especially MP6) mainly

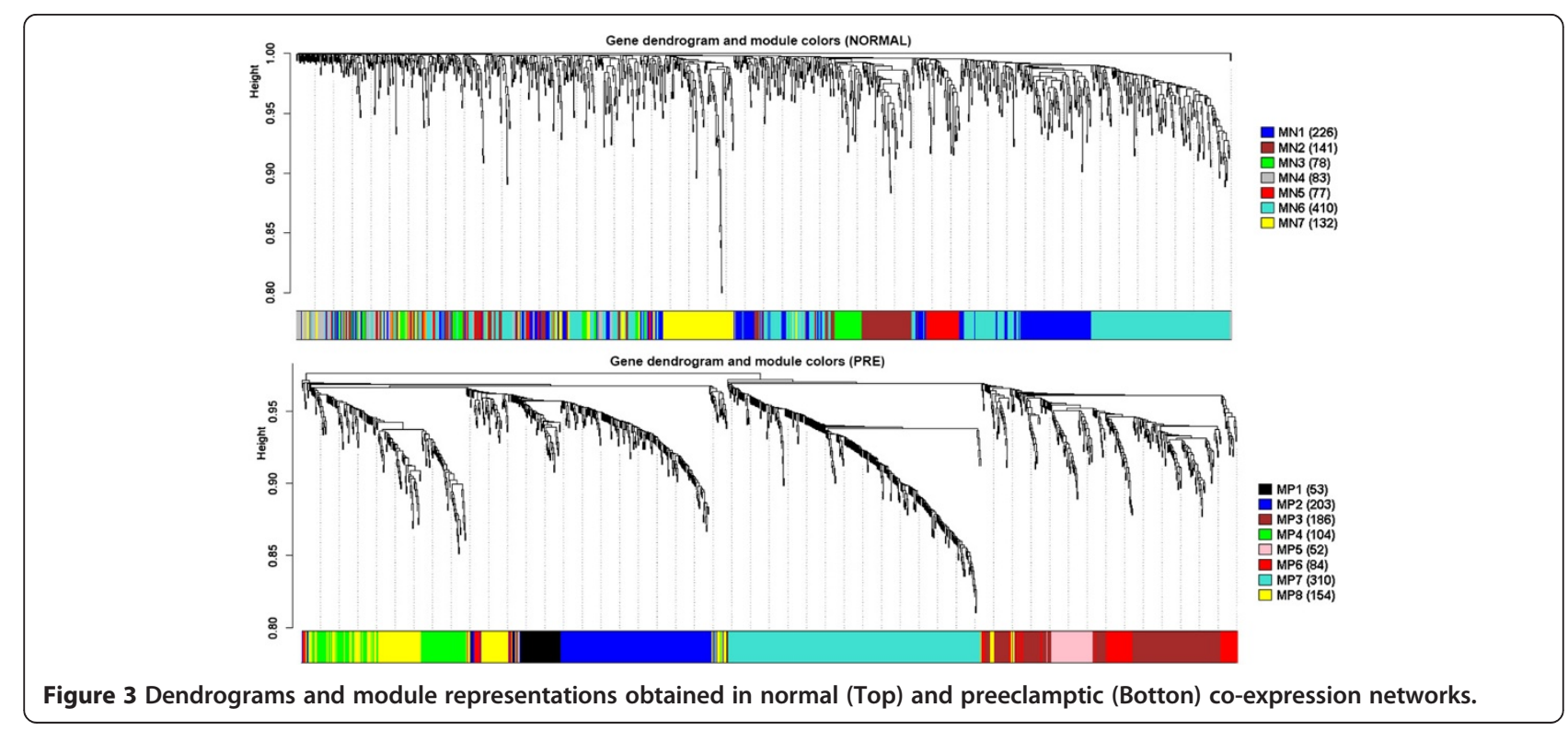




\begin{tabular}{l|c|c|c|c|c|c|c|}
\multicolumn{1}{c}{} & \multicolumn{1}{c}{ MN1 } & MN2 & MN3 & MN4 & MN5 & MN6 & MN7 \\
\cline { 2 - 8 } MP1 & 11 & 2 & 3 & 3 & - & 19 & 15 \\
\cline { 2 - 8 } MP2 & 84 & 8 & 2 & 8 & 3 & 94 & 4 \\
\cline { 2 - 8 } MP3 & 12 & 46 & 51 & 17 & 2 & 27 & 31 \\
\cline { 2 - 8 } MP4 & 14 & 11 & 4 & 19 & 30 & 17 & 9 \\
\cline { 2 - 8 } MP5 & 4 & 25 & 3 & - & - & 12 & 8 \\
\hline MP6 & 4 & 4 & 10 & 8 & 1 & 22 & 35 \\
\hline MP7 & 59 & 30 & 3 & 20 & 5 & 168 & 25 \\
\cline { 2 - 8 } MP8 & 37 & 15 & 2 & 8 & 36 & 51 & 5 \\
\cline { 2 - 8 } & & & & & & &
\end{tabular}

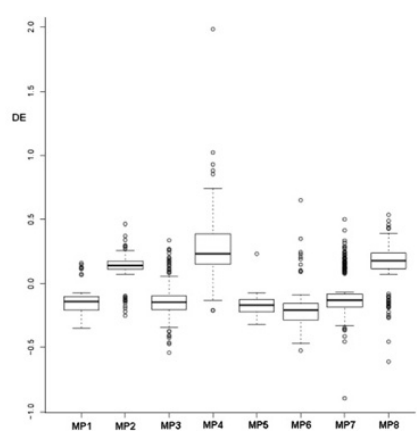

Figure 4 Left: Fisher's exact test in modules superposition where coloured cells represents p-value $<0.05$; Right: CoP modules differential expression (DE).

down-regulated genes. Genes in MP6 module enrich biological processes as (p-value $<0.01$ ): vascular development, blood vessel development, vasoconstriction, cell adhesion, regulation of endothelial cell proliferation as well as metabolic pathways ( -value $<0.01$ ) like ECMreceptor interactions and focal adhesion. All these processes are relevant and well known in PRE.

Besides considerations of expression values we must also consider the way in which genes are connected, actually, even when the modules overlap is significant, a different picture emerges by restricting the overlap to genes with increased intra-modular connectivity $\left.\left(k_{i}^{m}\right\rangle\left\langle k_{i}^{m}\right\rangle+\operatorname{std}\left(k_{i}^{m}\right)\right)$. Table 2 shows the top 10 genes with increased intra-modular connectivity (some modules do not have 10 tops and hence a reduced number is displayed), where we may notice that a few genes overlap between modules (only 28 genes of 145 selected higher). These results suggest that a large amount of common genes are not highly connected or, more importantly, they are highly related, but only in one of the networks.

MP7 module showed 168 significant overlapped genes with MN6. However, after the connectivity restriction few remained, even when both MP7 and MP6 are modules with higher intra-modular connectivity (Table 3). This suggests that MP7 could be a potential module for hubs identification. In addition, some of the selected genes, for example in MP4, are well known in relation with PRE (i.e. INHA, FLT, ENG see Discussion) and that are not shared with the CoN modules.

The variation of $\left\langle k_{i}^{m}\right\rangle$ tend to be higher than $<\mathrm{k}>$ and not necessarily expresses the same behaviour for both, i.e. the MP5 modules showed the highest $<\mathrm{k}>$ values but almost the minimal $\left\langle k_{i}^{m}\right\rangle$ ones. Modules with increased intra-modular connectivity suggest genes involved in similar process, however, in terms of significance, the modules (or differentiability) on the intra-modular connectivity cannot be considered without global connectivity. In terms of expression differentiability, MP4 module can be considered relevant because of the higher expression differences
(Figure 4 right) but considering the connectivity aspects we should include MP7 by the higher intra-modular connectivity. It is precisely this dichotomy between expression and connectivity that leads us to consider further based gene analysis.

The enrichment analysis for the MP5 and MP7 modules indicates less specific processes that can agree with the highest $<k>$ in both modules. The gene ontology database has a hierarchical structure where the specificity of the

\section{Table 2 Modular analysis}

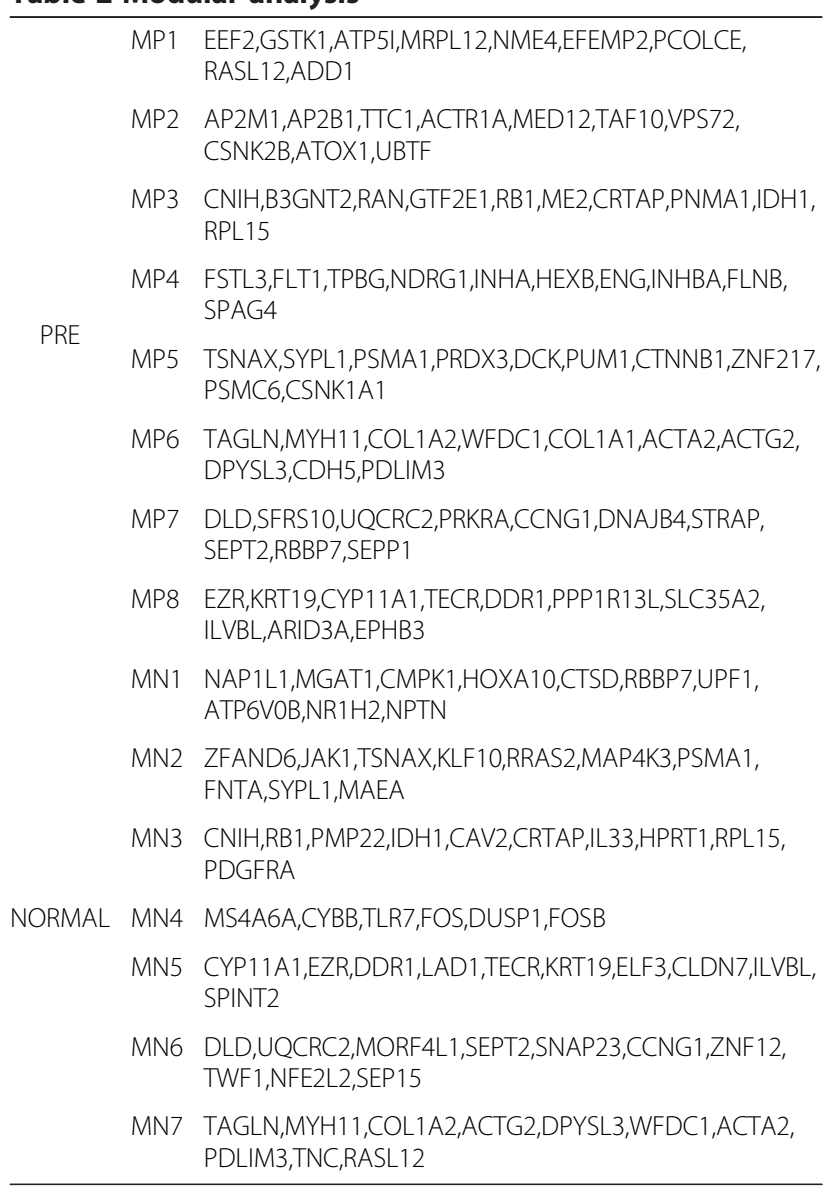

Selected genes with the highest intra-modular connectivity. 
Table 3 Modules statistics

\begin{tabular}{lccccc}
\hline CoP modules & $\langle\boldsymbol{k}\rangle$ & $\left\langle\mathbf{k}_{\mathbf{i}}^{\mathbf{m}}\right\rangle$ & CoN modules & $\langle\boldsymbol{k}\rangle$ & $\left\langle\mathbf{k}_{\mathbf{i}}^{\mathbf{m}}\right\rangle$ \\
\hline MP1 & 31.68 & 4.24 & MN1 & 1.75 & 0.83 \\
MP2 & 32.41 & 13.31 & MN2 & 1.25 & 0.58 \\
MP3 & 33.61 & 11.61 & MN3 & 0.95 & 0.29 \\
MP4 & 30.92 & 8.41 & MN4 & 0.14 & 0.02 \\
MP5 & 35.15 & 4.29 & MN5 & 1.08 & 0.39 \\
MP6 & 31.28 & 5.20 & MN6 & 2.29 & 1.62 \\
MP7 & 34.04 & 20.41 & MN7 & 0.95 & 0.49 \\
MP8 & 31.06 & 7.96 & & & \\
\hline
\end{tabular}

biological processes increases at each ramification level. Modules such as MP4 and MP6 previously discussed are significantly enriched in biological processes, even at level 4 or 5 leading us to specific (and therefore more relevant) processes, while a different pattern emerges from MP7 and MP5.

Most of the statistically significant biological processes enriched in MP5 module can't go further than level 2 (i.e., cell cycle process and organelle organization), and therefore is expected a high $\langle\mathrm{k}\rangle$ but lower $\left\langle k_{i}^{m}\right\rangle$ as shown in Table 3. Moreover, the genes in MP7 modules enrich a great number of processes in the level 1 and level 2, but also at level 4 and 5 likes (p-value $<0.01$ ): protein folding, DNA metabolic processes and protein transport. These processes related with MP7 module even when specifics are actually part of many central pathways and therefore we should expect a high $\langle\mathrm{k}\rangle$ and $\left\langle k_{i}^{m}\right\rangle$ as shown in Table 3.

Analysing nodes (genes) distance between the two networks (Figure 5), it can be noted that even for relatively low values of distance cut-offs (expressed as percentage of the maximum distance), the number of genes obtained were statistically significant ( $\mathrm{p}$-value $<0.01$ at $75 \%$ ) compared to the cut-off of the same randomized networks. For a cut-off distance $>85,90$, and 95\% we identified 261, 46 and 14 genes, respectively, distributed in several modules. As can be seen the representative modules according to KDist are the MP4 and MP7 considering the biggest differences between the node distances and also in correspondence with the previous modules analysis. In fact, in the 14 genes with maximum KDist values- NDRG1, FLT1, TPBG, FSTL3, FLNB, INHBA, SPAG4, INHA, HK2, HEXB, TPI1, BCL6, $L E P$, QSOX1- we also found FLT1, FLNB, INHA, LEP and $I N H B A$, which are some of the nodes with greater intramodular connectivity.

The analysis of genes based on GA algorithms also indicates a strong participation of MP4, but also including MP1 with GADA (Table 4). Moreover, we found at least one model with more than $97 \%$ of correct classification using the GADA procedure while all models revealed a LOO value higher than $90 \%$, with 30 selected genes. The MP1 inclusion as a relevant module is a result of expression exclusive analysis. This means, GA methods consider only the expression values in the model generation and selection of variables and therefore modules like

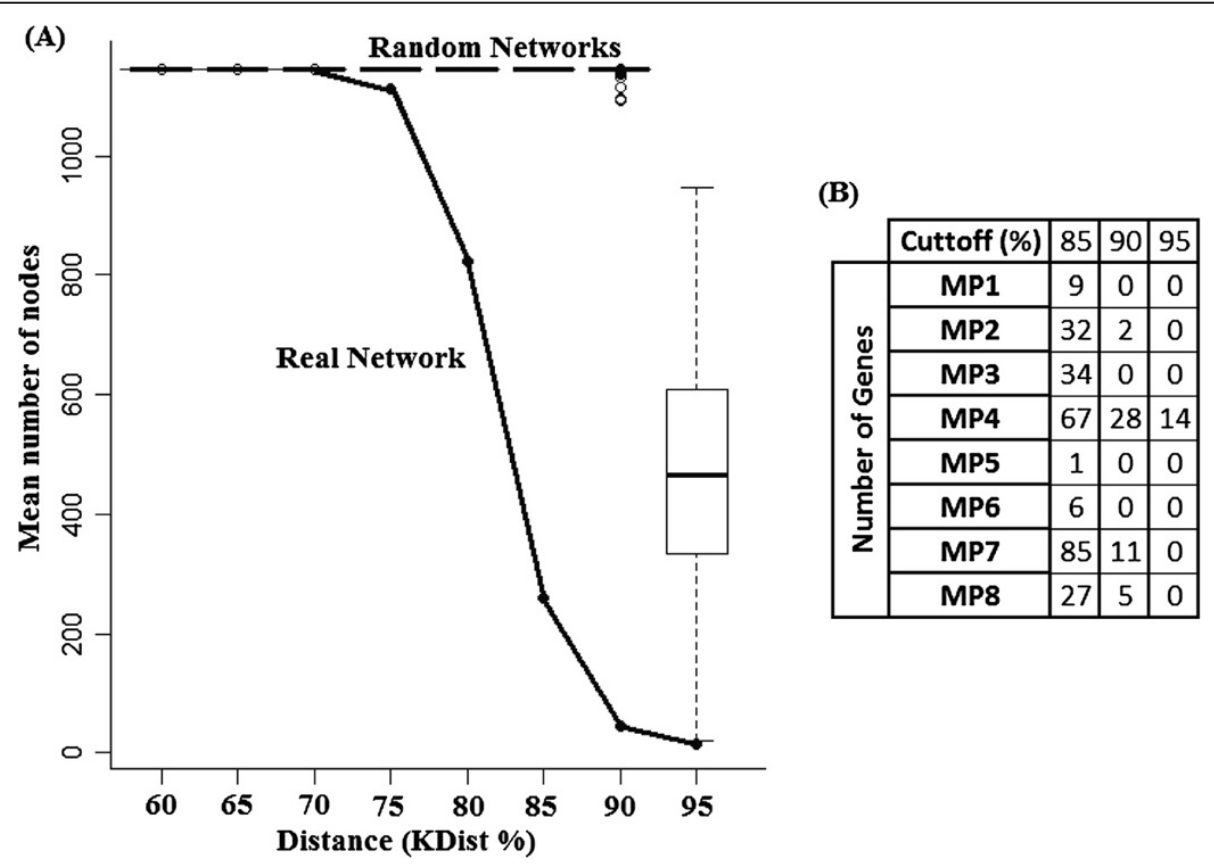

Figure 5 Distance cutoff analysis (A) and genes distribution across modules (B) for different cutoff values. The distance cutoff is expressed as the percent of the maximal distance. 
Table 4 Genetic algorithm methods results and genes distribution across PRE co-expression network modules

\begin{tabular}{|c|c|c|c|c|c|c|c|c|c|c|}
\hline Methods & $<\mathrm{LOO}>$ & $\mathrm{LOO}_{\text {max }}$ & MP1 & MP2 & MP3 & MP4 & MP5 & MP6 & MP7 & MP8 \\
\hline GANN & 0.940 & 0.965 & 0.92 & 1.06 & 1.07 & 1.13 & 1.11 & 0.91 & 0.91 & 0.98 \\
\hline GADA & 0.952 & 0.978 & 1.54 & 0.94 & 1.02 & 1.31 & 0.59 & 1.17 & 0.90 & 0.91 \\
\hline
\end{tabular}

Notes: LOO: leave-one-out cross-validation procedure; <LOO> corresponds to the average LOO cross-validation value in the 25 runs; $\mathrm{LOO}_{\text {max }}$ is the maximum $\mathrm{LOO}$ value obtained. The numbers in each of the CoP modules correspond to a ratio of modules that participate in the total 25 models.

MP7 with a higher intra-modular connectivity, but lower differential expression may be underestimated.

Analysing all the genes covered by the 25 models we have: 496 (GANN) and 337 (GADA). The overlap between the two methods led to 163 genes, but only 11 were also found with KDist>90\%: FLT1, TPBG, FLNB, INHBA, BCL6, QSOX1, HILPDA, ENG, PROCR, TTC1 and SLC6A8. Most of these genes belong to MP4 modules $(n=10)$, as expected, due to the influence of this module in expression values, however, being in the top list of KDist indicate that these genes also reveal some connectivity contribution. Therefore, these genes are an intermediate point comprising expression and connectivity however, must be plausible to consider the extremes genes in both connectivity (like INHA, NDRG1, FSTL3 and/or RBBP7) and expression differences (like $M M P 1, G C L M$, and/or RARRES2).

To facilitate further discussion we integrated the 163 genes obtained by GANN and GADA superposition in different graphical representations containing information about connectivity, KDist and expression (Figures 6 and 7).

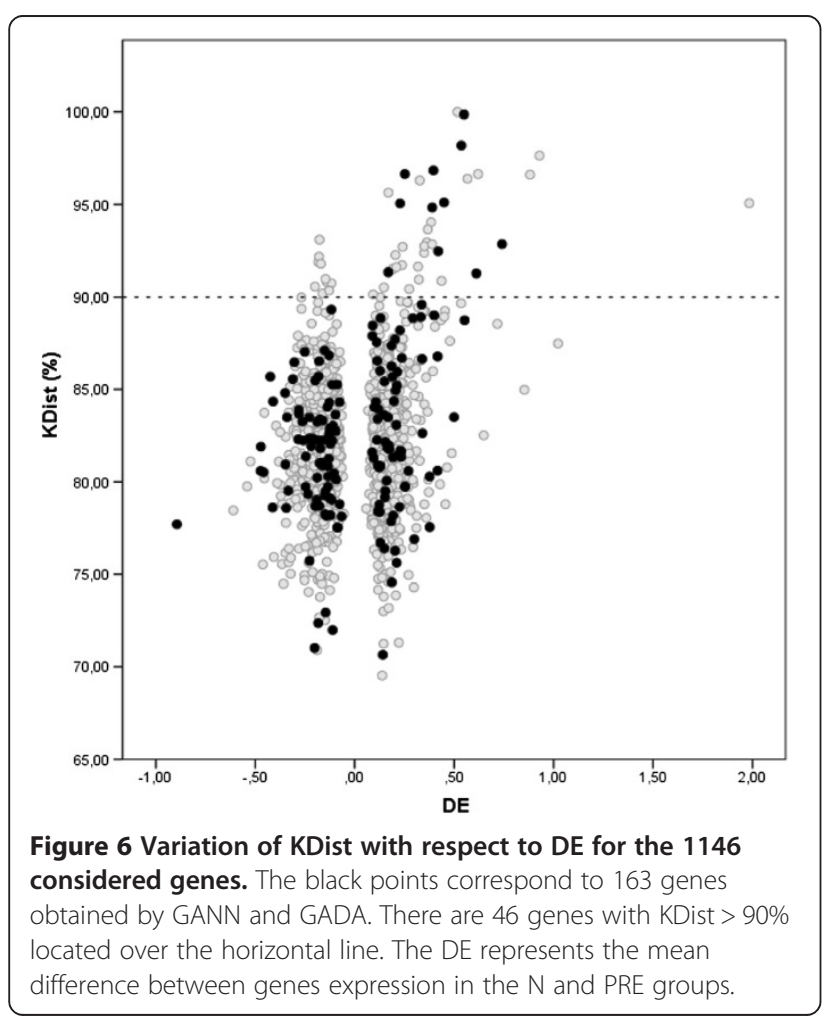

Figure 6 shows that the 163 genes obtained by GADA and GANN superposition actually cover a wide range of behaviours expression (down-regulated and up-regulated) and also some of the genes with the highest KDist value. Interestingly, the most down-regulated gene (MMP1) is covered by GA but not the up-regulated (LEP) one. We also can see that the highly up-regulated genes are not necessarily those with the highest topological differentiability. This pattern is even frequent with low regulated genes.

Figure 7 clearly shows that genes with higher intramodular connectivity are not necessarily those with large topological differences or prioritized by the procedures of genetic algorithms. Even when in CoN there is a clear trend toward for selecting genes with low intra-modular connectivity, in CoP there is no clear trend. Genes with KDist $>90 \%$ revealed a preference for a minimum intramodular connectivity in $\mathrm{N}$ network but not the maximum in the PRE network.

\section{Discussion}

The lower genes connectivity correlation instead of gene expression intensity between $\mathrm{N}$ and PRE groups indicates that genes expression profile is similar in both situations (also partially supported by the similarities between network modules). This means that genes with high or low expression in $\mathrm{N}$ are also high or low expressed in PRE. However, the mode by which genes are interconnected or correlated is not so conserved. This also suggests that we have useful information for differentiating normal vs disease by analysing the connectivity network as a complement to an exclusive analysis of genes expression intensity. Furthermore, the increased connectivity degree in the network of PRE group can be a reflection of a highly systemic disorder involving multiple metabolic pathways. This could justify the high probability of multifactorial causes and also the possibility of preeclampsia to progress through more complex clinical conditions [33].

The comparison of network modules between the two conditions revealed that the genes were grouped similarly. All modules revealed some similarities in genes composition between the two conditions. However, there are main differences shown by the way (and strength) by which they are connected. In this regard, the MP7 module is highly similar to MN6 sharing several genes, and many of them also with a high degree of connectivity (i.e. $D L D$ and UQCRC2) (see Table 2 and Figure 4 left), but it is still more similar to MP6 and MN7 modules sharing a smaller 

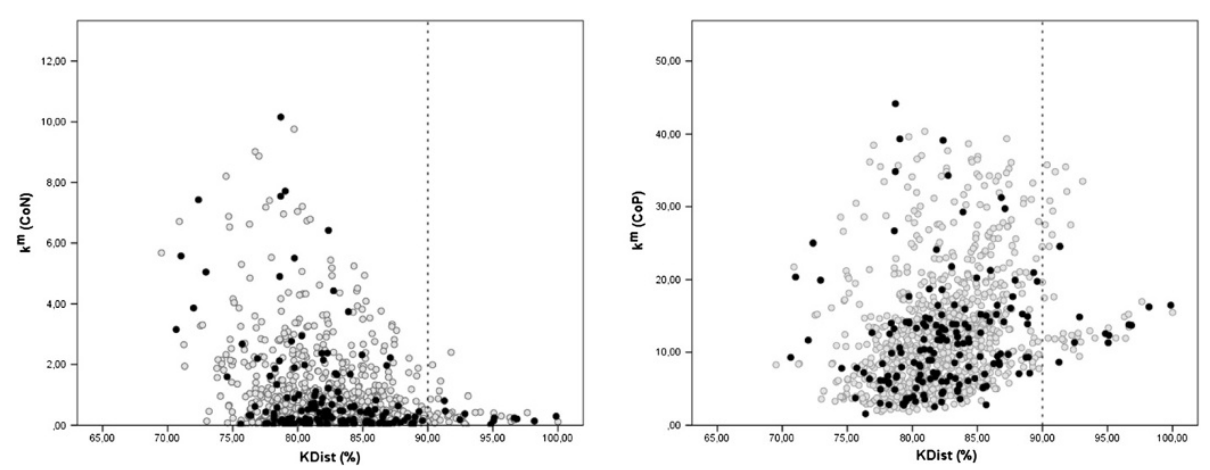

Figure $7 \mathrm{KDist}$ variation with respect to gene intra-modular connectivity (Left) in normal network (CoN) and (Right) in PRE network (CoP) for the 1146 considered genes. The black points correspond to 163 genes obtained by GANN and GADA. There are 46 genes with KDist $>90 \%$ cuttof marked by the broken line.

number of genes, but most of them with highest connectivity values (see Table 2 and Figure 4 Left). The average connectivity (total or intra-modular) indicates that MP7 contain genes with strong inter-correlations, however, as we noticed, intra-modular connectivity itself could have misled the module selection in terms of prioritization.

Our results based on KDist together with modular analysis indicate that MP4 and MP7 modules comprise the genes with the greatest potential differentiability (Figure 6 Left). In the group of 14 genes with KDist $>95 \%$ (all of them in the module MP4) we found FLT1, LEP, INHA and FSTL3. These genes are well known related to preeclampsia [34-43] and in fact FLT1 and INHA have been used as potential early predictive markers in multivariate models [41-43]. Inhibin B (INHB), however, has not been studied, as well as INHA during preeclampsia or general pregnancy hypertension and only a previous study indicating significant differences in preeclampsia was found [44]. A similar situation was observed for BCL6 [45]. Previous publications support an up-regulation of TPBG [46] and also $N D R G 1$ [47]. However, these genes have not been well studied during pregnancy. Other genes as SPAG4, HEXB, TPI1 and QSOX1 are basically unknown in preeclampsia (and even during normal pregnancy). Interestingly, of the 50 genes previously identified in another study and using other network approaches [8], 11 genes were consistent with our KDist $>90 \%$, including the little exploited TPBG, NDRG1, BCL6, LYN and FLNB. Several of these proteins are related to hormone/endocrine pathways (ie, $L E P$, $I N H A, I N H B)$, a significant process already highlighted by the enrichment analysis.

The genetic algorithm procedures have led to a high number of genes mainly by using GANN. The nearest neighbour is a non-linear method; therefore, it was not influenced by the variables distribution. However, it is probably by the nearest neighbour approach to select genes that are highly significant (in terms of classification) as a group rather than as individuals [48].
Therefore, given the high co-linearity between genes expression, is quite possible to select a wide range of genes combination capable of achieving a good classification and thus considerably increase the gene space through different models [48]. Even when the best models were obtained with GADA, we think that the main advantage was not in LOO values but in the reduced gene space compared to GANN. On the other hand, the mathematical bases of nearest neighbour classifications are quite different from discriminant analysis. Therefore, the overlapping of both genes spaces, even when functional, may be a drastic approach with risks of rejecting significant genes detected by the methods in their own model space.

Both of these methods GANN and GADA share a large number of genes (163) and some (11) are also present in the KDist $>90 \%$ group. In this reduced group we found again FLT1, TPBG, FLNB, INHBA, BCL6 and QSOX1 discussed above, but also other genes such as ENG and PROCR [34-43,49] which are well documented in preeclampsia. Interestingly some of these genes (i.e. FLT1, FLNB and ENG) are well known related with signalling pathways involved in cytokines interactions and angiogenesis according to the biological processes and pathways enrichment analysis.

These results suggest that the consideration of distance based on the co-expression network connectivity is promising for the identification of significant genes; however, a cutoff of $95 \%$ excludes relevant genes. This can be observed not only by the inclusion of genes obtained by GA in the $90 \%$ cutoff but also by looking other MP4 genes contained in the range of $90 \%$. In this group we have, for example, the HTRA1 and ACVR1 that have been well documented in preeclampsia [50-52].

The genes obtained by GA procedures appear to complement those obtained by KDist cutoff $>90 \%$ in several ways. Figures 6 and 7 suggest that highly up-regulated genes are better resolved by KDist or general topological 
measurements, contrary to the down-regulated genes. This preference of up-regulated gene to be highly focused was reported by other authors, considering the network interactions [53,54]. However, some of the down-regulated genes, uniquely identified by GA procedures are well documented in studies during PRE as XBP1 and MMP1 [55-57] and thus can also be significant to the understanding of the disease and its characterization.

Our results indicate that co-expression network analysis combining both modular and gene centred approaches are capable to identify genes significantly related to preeclampsia. Some of these genes are consistent through genetic algorithms approaches where other down-regulated genes were relevantly prioritized. We were able to corroborate some of the identified genes through manually literature revision in order to validate the hubs identification. However, some other genes remain unexplored or unknown, not only in preeclampsia, but also during pregnancy, leading to the need of further experimental confirmation.

\section{Conclusions}

Genes in weighted co-expression network revealed a similar distribution between detected modules in N and PRE conditions. However, major differences were obtained considering the connectivity of nodules. Genes with more connectivity or intra-modular connectivity were not always detected as network hubs and better results were obtained by comparing the gene and its neighbourhood between the two conditions. In addition, all models obtained by genetic algorithms were consistent with a successful classification higher than $90 \%$, restricting the 30 variables to at least one model greater than $95 \%$.

Gene prioritization from microarray data was improved considering both, gene expression and genes co-expression (connectivity) information. In this sense the co-expression weighted network and genetic algorithms clearly provided consistent and complementary results. Combining the two methods we identified, it is well known preeclampsia related genes like: FLT1, LEP, INHA, ENG, PROCR, MMP1, $X B P 1$ and FSTL3. However, other genes as FLNB, INHBA, $B C L 6, T P B G, N D R G 1, L Y N$ and QSOX1 were also significant in our analysis, but this has been little explored or is unknown in the current state of the art of preeclampsia pathophysiology. Therefore, these results indicate that more experimental research is warranted to exploit the role of these genes in the development of pregnancy.

\section{Competing interests}

The authors declare that they have no competing interests.

\section{Authors' contributions}

All authors participated in the study design and coordination. ET performed the network and statistical analysis, as well as drafted the manuscript. JB and IR participated in the discussion of the results as well as the final writing and correction. All authors read and approved the final manuscript.

\section{Acknowledgements}

This study was supported by "Fundação para a Ciência e a Tecnologia" (FCT), grant: SFRH/BPD/72391/2010.

\section{Author details}

${ }^{1}$ Institute for Molecular and Cell Biology (IBMC), University of Porto, Porto, Portugal. ${ }^{2}$ Center for Research in Health Technologies and Information Systems (CINTESIS), Faculty of Medicine, University of Porto, Porto, Portugal. ${ }^{3}$ Department of Obstetrics and Gynecology, São João Hospital of Porto; INEB - Institute of Biomedical Engineering, Porto, Portugal. ${ }^{4}$ Laboratory of Biochemistry, Department of Biological Sciences, Faculty of Pharmacy, University of Porto, Porto, Portugal. ${ }^{5}$ Institute for Molecular and Cell Biology (IBMC), University of Porto, Portugal, Porto, Portugal.

Received: 26 March 2013 Accepted: 8 November 2013 Published: 12 November 2013

\section{References}

1. Leeman L, Fontaine P: Hypertensive disorders of pregnancy. Am Fam Physician 2008, 78(1):93-100.

2. National Collaborating Centre for Women's and Children's Health: Hypertension in pregnancy. The management of hypertensive disorders during pregnancy. London (UK): National Institute for Health and Clinical Excellence (NICE); 46. Clinical guideline; no. 107; 2010.

3. Jeffcoate TN: Pre-eclampsia and eclampsia: the disease of theories. Proc $R$ Soc Med 1966, 59(5):397-404.

4. Sheppard SJ, Khalil RA: Risk factors and mediators of the vascular dysfunction associated with hypertension in pregnancy. Cardiovasc Hematol Disord Drug Targets 2010, 10(1):33-52.

5. Nishizawa H, Pryor-Koishi K, Kato T, Kowa H, Kurahashi H, Udagawa Y: Microarray analysis of differentially expressed fetal genes in placental tissue derived from early and late onset severe pre-eclampsia. Placenta 2007, 28(5-6):487-497.

6. Founds SA, Conley YP, Lyons-Weiler JF, Jeyabalan A, Hogge WA, Conrad KP: Altered global gene expression in first trimester placentas of women destined to develop Preeclampsia. Placenta 2009, 30(1):15-24.

7. Sitras V, Paulssen RH, Grønaas H, Leirvik J, Hanssen TA, Vårtun A, Acharya G: Differential placental gene expression in severe preeclampsia. Placenta 2009, 30(5):424-433.

8. Tejera E, Bernardes J, Rebelo I: Preeclampsia: a bioinformatics approach through protein-protein interaction networks analysis. BMC Syst Bio/ 2012, 6:97.

9. Barrett T, Troup DB, Wilhite SE, Ledoux P, Evangelista C, Kim IF, Tomashevsky M, Marshall KA, Phillippy KH, Sherman PM, Muertter RN, Holko M, Ayanbule O, Yefanov A, Soboleva A: NCBI GEO: archive for functional genomics data sets-10 years on. Nucleic Acids Res 2011, 39:D1005-D1010.

10. Parkinson H, Sarkans U, Kolesnikov N, Abeygunawardena N, Burdett T, Dylag M, Emam I, Farne A, Hastings E, Holloway E, Kurbatova N, Lukk M, Malone J, Mani R, Pilicheva E, Rustici G, Sharma A, Williams E, Adamusiak T, Brandizi M, Sklyar N, Brazma A: ArrayExpress update-an archive of microarray and high-throughput sequencing-based functional genomics experiments. Nucleic Acids Res 2011, 39:D1002-D1004.

11. Roten LT, Johnson MP, Løset M, Mundal SV, Forsmo S, Skorpen F, Fenstad MH, Dyer TD, Blangero J, Moses EK, Austgulen E: Evaluation of COMT as a maternal preeclampsia candidate susceptibility gene; assessed by genotyping of the Val158Met polymorphism and by transcriptinal profiling of decidual tissue. Array Express Database 2011. Ref:E-TABM-682. Last update, June 2011.

12. Eide IP, Isaksen CV, Salvesen KA, Langaas M, Schønberg SA, Austgulen R: Decidual expression and maternal serum levels of heme oxygenase 1 are increased in pre-eclampsia. Acta Obstet Gynecol Scand 2008, 87(3):272-279.

13. Tsai S, Hardison NE, James AH, Motsinger-Reif AA, Bischoff SR, Thames BH, Piedrahita JA: Transcriptional profiling of human placentas from pregnancies complicated by preeclampsia reveals disregulation of sialic acid acetylesterase and immune signalling pathways. Placenta 2011, 32(2):175-182.

14. Winn VD, Gormley M, Paquet AC, Kjaer-Sorensen K, Kramer A, Rumer KK, Haimov-Kochman R, Yeh RF, Overgaard MT, Varki A, Oxvig C, Fisher SJ: Severe preeclampsia-related changes in gene expression at the maternal-fetal interface include sialic acid-binding immunoglobulin-like lectin-6 and pappalysin-2. Endocrinology 2009, 150(1):452-462.

15. Gautier L, Cope L, Bolstad BM, Irizarry RA: affy--analysis of Affymetrix GeneChip data at the probe level. Bioinformatics 2004, 20(3):307-315. 
16. Gentleman RC, Carey VJ, Bates DM, Bolstad B, Dettling M, Dudoit S, Ellis B, Gautier L, Ge Y, Gentry J, Hornik K, Hothorn T, Huber W, lacus S, Irizarry R, Leisch F, Li C, Maechler M, Rossini AJ, Sawitzki G, Smith C, Smyth G, Tierney L, Yang JY, Zhang J: Bioconductor: open software development for computational biology and bioinformatics. Genome Biol 2004, 5(10):R80

17. Du P, Kibbe WA, Lin SM: lumi: a pipeline for processing Illumina microarray. Bioinformatics 2008, 24(13):1547-1548.

18. Davis S, Meltzer PS: GEOquery: a bridge between the Gene Expression Omnibus (GEO) and BioConductor. Bioinformatics 2007, 23(14):1846-1847.

19. Fan $X$, Shao L, Fang $H$, Tong W, Cheng Y: Cross-platform comparison of microarray-based multiple-class prediction. PLoS One 2011, 6(1):e16067.

20. Sîrbu A, Ruskin HJ, Crane M: Cross-platform microarray data normalisation for regulatory network inference. PLoS One 2010, 5(11):e13822.

21. Rudy J, Valafar F: Empirical comparison of cross-platform normalization methods for gene expression data. BMC Bioinforma 2011, 12:467.

22. Du P, Kibbe WA, Lin SM: nulD: a universal naming scheme of oligonucleotides for illumina, affymetrix, and other microarrays. Biol Direct 2007, 2:16

23. Carlson M: hgu133b.db: Affymetrix Human Genome U133 Set annotation data (chip hgu133b). R package version 2.8.0.

24. Langfelder $\mathrm{P}$, Horvath S: WGCNA: an R package for weighted correlation network analysis. BMC Bioinforma 2008, 9:559.

25. Miller JA, Cai C, Langfelder P, Geschwind DH, Kurian SM, Salomon DR, Horvath S: Strategies for aggregating gene expression data: the collapseRows R function. BMC Bioinforma 2011, 12:322.

26. Johnson WE, Li C, Rabinovic A: Adjusting batch effects in microarray expression data using empirical Bayes methods. Biostatistics 2007, 8:118-127.

27. Smyth GK: Limma: linear models for microarray data. In Bioinformatics and Computational Biology Solutions using $R$ and Bioconductor. Edited by Gentleman R, Carey V, Dudoit S, Irizarry R, Huber W. New York: Springer; 2005

28. Zhang $B$, Horvath $S$ : A general framework for weighted gene co-expression network analysis. Stat App/ Genet Mo B 2005, 4(1):17.

29. Langfelder $P$, Zhang $B$, Horvath S: Defining clusters from a hierarchical cluster tree: the Dynamic Tree Cut package for R. Bioinformatics 2007, 24(5):719-720.

30. Ray M, Yunis $R$, Chen $X$, Rocke DM: Comparison of low and high dose ionising radiation using topological analysis of gene coexpression networks. BMC Genomics 2012, 13:190.

31. Ray $M$, Zhang $W$ : Analysis of Alzheimer's disease severity across brain regions by topological analysis of gene co-expression networks. BMC Syst Biol 2010, 4:136.

32. Huang DW, Sherman BT, Lempicki RA: Systematic and integrative analysis of large gene lists using DAVID Bioinformatics Resources. Nature Protoc 2009, 4(1):44-57.

33. Powe CE, Levine RJ, Karumanchi SA: Preeclampsia, a disease of the maternal endothelium. Circulation 2011, 123:2856-2869.

34. McElrath TF, Lim KH, Pare E, Rich-Edwards J, Pucci D, Troisi R, Parry S: Longitudinal evaluation of predictive value for preeclampsia of circulating angiogenic factors through pregnancy. Am J Obstet Gynecol 2012, 207(5):407.e1-7.

35. Masoura S, Kalogiannidis IA, Gitas G, Goutsioulis A, Koiou E, Athanasiadis A, Vavatsi N: Biomarkers in pre-eclampsia: a novel approach to early detection of the disease. J Obstet Gynaecol 2012, 32(7):609-616.

36. Wang S, Qiao FY, Feng L: High leptin level and leptin receptor Lys656Asn variant are risk factors for preeclampsia. Genet Mol Res 2013, 4:12.

37. Hogg K, Blair JD, von Dadelszen P, Robinson WP: Hypomethylation of the LEP gene in placenta and elevated maternal leptin concentration in early onset pre-eclampsia. Mol Cell Endocrinol 2013, 367(1-2):64-73.

38. Guo J, Tian T, Lu D, Xia G, Wang H, Dong M: Alterations of maternal serum and placental follistatin-like 3 and myostatin in pre-eclampsia. J Obstet Gynaecol Res 2012, 38(7):988-996.

39. Founds SA, Terhorst LA, Conrad KP, Hogge WA, Jeyabalan A, Conley YP: Gene expression in first trimester preeclampsia placenta. Biol Res Nurs 2011, 13(2):134-139.

40. Pryor-Koishi K, Nishizawa H, Kato T, Kogo H, Murakami T, Tsuchida K, Kurahashi $H$, Udagawa Y: Overproduction of the follistatin-related gene protein in the placenta and maternal serum of women with pre-eclampsia. BJOG 2007, 114(9):1128-1137.

41. Rohra DK, Zeb A, Qureishi RN, Azam SI, Khan NB, Zuberi HS, Sikandar R: Prediction of pre-eclampsia during early pregnancy in primiparas with soluble fms-like tyrosine kinase- 1 and placental growth factor. Natl Med J India 2012, 25(2):68-73.
42. Aquilina J, Thompson O, Thilaganathan B, Harrington K: Improved early prediction of pre-eclampsia by combining second-trimester maternal serum inhibin-A and uterine artery Doppler. Ultrasound Obstet Gynecol 2001, 17(6):477-484.

43. Kuc S, Wortelboer EJ, van Rijn BB, Franx A, Visser GH, Schielen PC: Evaluation of 7 serum biomarkers and uterine artery Doppler ultrasound for first-trimester prediction of preeclampsia: a systematic review. Obstet Gynecol Surv 2011, 66(4):225-239.

44. Petraglia F, Luisi S, Benedetto C, Zonca M, Florio P, Casarosa E, Volpe A, Bernasconi S, Genazzani AR: Changes of dimeric inhibin B levels in maternal serum throughout healthy gestation and in women with gestational diseases. J Clin Endocrinol Metab 1997, 82(9):2991-2995.

45. Nishizawa H, Ota S, Suzuki M, Kato T, Sekiya T, Kurahashi H, Udagawa Y: Comparative gene expression profiling of placentas from patients with severe pre-eclampsia and unexplained fetal growth restriction. Reprod Biol Endocrinol 2011, 9:107.

46. Okazaki S, Sekizawa A, Purwosunu Y, Farina A, Wibowo N, Okai T: Placenta-derived, cellular messenger RNA expression in the maternal blood of preeclamptic women. Obstet Gynecol 2007, 110(5):1130-1136.

47. Choi SJ, Oh SY, Kim JH, Sadovsky Y, Roh CR: Increased expression of $\mathrm{N}$-myc downstream-regulated gene 1 (NDRG1) in placentas from pregnancies complicated by intrauterine growth restriction or preeclampsia. Am J Obstet Gynecol 2007, 196(1):45.e1-7.

48. Li L, Weinberg RC: Gene selection and sample classification using a genetic algorithm and k-Nearest neighbor method. In A Practical Approach to Microarray Data Analysis. Edited by Berrar DP, Dubitzky W, Granzow M. Kluwer Academic Publishers; 2003:216-229.

49. Saposnik B, Peynaud-Debayle E, Stepanian A, Baron G, Simansour M, Mandelbrot L, de Prost D, Gandrille S: Elevated soluble endothelial cell protein $C$ receptor (sEPCR) levels in women with preeclampsia: a marker of endothelial activation/damage? Thromb Res 2012, 129(2):152-157.

50. Marzioni D, Lorenzi T, Altobelli E, Giannubilo SR, Paolinelli F, Tersigni C Crescimanno C, Monsurrò V, Tranquilli AL, Di Simone N, Castellucci M: Alterations of maternal plasma HTRA1 level in preeclampsia complicated by IUGR. Placenta 2012, 33(12):1036-1038.

51. Yu L, Li D, Liao QP, Yang HX, Cao B, Fu G, Ye G, Bai Y, Wang H, Cui N, Liu M, Li YX, Li J, Peng C, Wang YL: High levels of activin A detected in preeclamptic placenta induce trophoblast cell apoptosis by promoting nodal signaling. J Clin Endocrinol Metab 2012, 97(8):E1370-E1379.

52. Akolekar R, Etchegaray A, Zhou Y, Maiz N, Nicolaides KH: Maternal serum activin a at 11-13 weeks of gestation in hypertensive disorders of pregnancy. Fetal Diagn Ther 2009, 25(3):320-327.

53. Jonsson PF, Bates PA: Global topological features on cancer proteins in the human interactome. Bioinformatics 2006, 22:2291-2297.

54. Wachi S, Yoneda K, Wu R: Interactome-transcriptome analysis reveals high centrality of genes differentially expressed in lung cancer tissue. Bioinformatics 2005, 21:4205-4208.

55. Lian IA, Toft JH, Olsen GD, Langaas M, Bjørge L, Eide IP, Børdahl PE, Austgulen $R$ : Matrix metalloproteinase 1 in pre-eclampsia and fetal growth restriction: reduced gene expression in decidual tissue and protein expression in extravillous trophoblasts. Placenta 2010, 31(7):615-620.

56. Mousa AA, Cappello RE, Estrada-Gutierrez G, Shukla J, Romero R, Strauss JF 3rd, Walsh SW: Preeclampsia is associated with alterations in DNA methylation of genes involved in collagen metabolism. Am J Pathol 2012, 181(4):1455-1463.

57. Lian IA, Løset M, Mundal SB, Fenstad MH, Johnson MP, Eide IP, Bjørge L, Freed KA, Moses EK, Austgulen R: Increased endoplasmic reticulum stress in decidual tissue from pregnancies complicated by fetal growth restriction with and without pre-eclampsia. Placenta 2011, 32(11):823-829.

doi:10.1186/1755-8794-6-51

Cite this article as: Tejera et al:: Co-expression network analysis and genetic algorithms for gene prioritization in preeclampsia. BMC Medical Genomics 2013 6:51. 